\title{
筷筋约束混凝土方柱轴压破坏尺寸效应细观数值 研究
}

杜敏 ${ }^{1,2^{*}}$, 金汶 $^{2}$, 杜修力 ${ }^{2}$, 李冬 ${ }^{2}$, 张帅 $^{2}$

1. 防灾科技学院防灾工程系, 三河 065201;

2. 北京工业大学城市减灾与防灾防护教育部重点实验室, 北京 100124

*E-mail:dumin2001@163.com

收稿日期: 2017-07-21; 接受日期: 2017-07-26; 网络出版日期: 2017-09-15

国家自然科学基金(批准号: 51408127)、国家自然科学基金创新研究群体项目(编号: 51421005)和防灾科技学院教育研究与教学改革项目 (编号: JY2016B07)资助

\begin{abstract}
摘要钢筋混凝土构件尺寸效应行为源于混凝土材料的非均质性及钢筋/混凝土复杂的非线性相互作用. 通 过建立反映上述特征的约束混凝土方柱3D细观数值模型, 开展轴心受压破坏行为及尺寸效应数值研究, 探讨 体积配䇽率对䇫筋约束混凝土方柱轴心受压力学行为及尺寸效应的影响机理, 并与试验结果进行对比分析. 基 于Bažant尺寸效应律, 开展约束混凝土轴心抗压强度尺寸效应理论分析. 研究结果表明: 数值模拟与试验结果 吻合良好, 构建的钢筋混凝土柱细观力学模型能够准确地描述筴筋约束混凝土构件的破坏行为及尺寸效应规 律; 体积配䇽率增加, 约束混凝土柱的名义强度增大、破坏呈现更少的脆性, 尺寸效应现象减弱; Bažant尺寸效 应理论能够对约束混凝土柱轴心加载下的尺寸效应行为做出合理解释.
\end{abstract}

关键词䇚筋约束, 混凝土方柱, 轴压强度, 尺寸效应, 配䇚率, 细观数值模拟

\section{1 引言}

约束混凝土是指利用外部约束, 改善混凝土自身 原有的受压特性, 以提高其抗压强度及变形能力 ${ }^{[1]}$. 约 束混凝土构件(包括: 䈐筋, FRP, CFRP及钢管等约束 方式)被广泛应用于土木工程. 约束混凝土材料的力 学行为是现代结构工程界研究的热点问题之一, 国内 外学者对此进行了大量的研究.

1903年, Considere指出螺旋箱筋的约束作用对混 凝土强度的提高具有积极作用 ${ }^{[2]}$. 1928年 Richart等
人 $^{[3]}$ 定量研究了液体围压对混凝土圆柱体轴压性能的 影响, 并提出约束混凝土抗压强度以及峰值应变的计 算理论. 1967年, Soliman和 $\mathrm{Yu}^{[4]}$ 开展了方形箨筋约束 混凝土试验研究, 考虑了䈌筋间距、直径、体积配䇽 率、混凝土全截面面积与受约束混凝土核心面积之 比等参数, 试验结果表明矩形箱筋约束也能够提高混 凝土的宏观强度. 1988年Mander等人 ${ }^{[5]}$ 基于钢筋混凝 土柱的试验结果, 提出了约束混凝土力学模型, 该模型 力学机理清晰、适用性广, 得到广泛应用. 此外, 钱稼 茹等人 ${ }^{[6]}$ 、史庆轩等人 ${ }^{[1,7]}$ 、郑文忠和侯羽驰 ${ }^{[8]}$ 及West

引用格式: 杜敏, 金浏, 杜修力, 等. 筢筋约束混凝土方柱轴压破坏尺寸效应细观数值研究. 中国科学: 技术科学, 2017, 47: 1057-1066

Du M, Jin L, Du X L, et al. Mesoscopic simulation of size effect on stirrup-confined concrete columns under axial compression (in Chinese). Sci Sin Tech, 2017, 47: 1057-1066, doi: 10.1360/N092017-00240 
等人 ${ }^{[9]}$ 亦对箍筋约束混凝土的承载力计算方法进行了 深入的研究, 他们提出的理论方法计算结果与试验结 果(较小尺寸试件试验结果)吻合很好. 这些研究工作 促进了对箍筋约束混凝土力学性能的认识. 纵使如此, 现有䇽筋约束混凝土应力-应变本构关系模型以及我 国《混凝土结构设计规范》 ${ }^{[10]}$ 是基于缩尺模型研究 成果提出, 未考虑尺寸效应对约束混凝土柱轴心受压 承载力的影响.

大量试验工作, 如文献[11 16]证实了䈨筋约束混 凝土柱在轴心加载下的承载力(或名义轴压强度)具有 尺寸效应特征. 这些试验工作主要针对小尺寸箍筋约 束混凝土柱. 受到试验设备与经济条件的限制, 对大 尺寸箱筋约束混凝土柱的轴压破坏行为及尺寸效应 的研究很少涉及.

钢筋混凝土构件尺寸效应源于两个方面: (1) 混 凝土材料本身的尺寸效应——非均质性; (2) 钢筋/混 凝土复杂的非线性相互作用. 因此, 如果能够建立合 理的钢筋混凝土构件的数值分析模型, 反映上述两方 面的全部“特征”, 即可采用有限元数值模拟手段, 研究 并揭示钢筋混凝土构件宏观力学特性及其破坏行为, 拓展试验研究的尺度范围, 对大尺寸约束混凝土构件 的力学特性和破坏行为进行预测.

一些研究者尝试采用细观力学分析方法来模拟 钢筋混凝土构件破坏行为及尺寸效应问题, 如Rios和 Riera $^{[17]}$ 基于二维离散元模型, 考虑混凝土细观结构形 式, 数值模拟了钢筋混凝土梁名义强度的尺寸效应行 为; Wang ${ }^{[18]}$ 基于二维细观尺度分析方法(RBSM法)模 拟了钢筋混凝土梁的破坏行为. $\mathrm{Du}$ 等人 ${ }^{[19]}$ 尝试将细观 力学分析方法扩展到三维情况, 基于具有较高计算效 率的细观单元等效化模型与分析方法, 建立了钢筋混 凝土柱尺寸效应研究的三维细观尺度分析模型, 初步 探讨了钢筋混凝土柱在轴压荷载作用下的尺寸效应 行为. 总体来说, 由于计算量庞大、运算效率低等原 因, 约束混凝土构件尺寸效应的细观数值研究工作主 要集中于二维模型, 使用三维细观数值模型开展研究 较为少见.

鉴于此, 本文拟开展约束混凝土构件尺寸效应行 为的数值理论研究, 从细观尺度出发假定混凝土由 骨料颗粒、砂浆基质、界面过渡区(ITZ)组成, 采用 钢筋-混凝土黏结滑移本构关系模型来描述其相互作 用, 建立钢筋混凝土柱 $3 \mathrm{D}$ 细观数值模型, 开展构件截
面尺寸在267 800 mm, 长细比为 3 , 体积配䈐率为 $0 \%$, $1.26 \%, 2.89 \%$ 的箱筋约束混凝土柱轴心受压加载数值 实验, 探讨体积配䈨率对䈐筋约束混凝土方柱尺寸 效应的影响机理, 并与试验结果进行对比分析. 基于 Bažant尺寸效应理论对约束混凝土构件轴心加载下的 尺寸效应行为开展理论分析.

\section{2 约束混凝土柱细观尺度力学模型}

如前所述, 钢筋混凝土构件尺寸效应源于混凝土 材料本身的尺寸效应——非均质性, 以及钢筋/混凝土 复杂的非线性相互作用. 因此, 合理的钢筋混凝土构 件数值分析模型不仅能够表征混凝土材料本身的尺 寸效应, 还应准确地描述钢筋/混凝土的非线性相互作 用行为. 最好的方法是采用能精准描述混凝土细观结 构形式的细观尺度数值分析方法 ${ }^{[20]}$.

\section{1 筷筋约束混凝土柱微细观结构}

在细观层次上假定混凝土材料由骨料颗粒、砂 浆介质和界面过渡区组成, 骨料颗粒假定为球体, 采 用 30 和 $16 \mathrm{~mm}$ 的等效粒径表示骨料颗粒的连续级配, 骨料含量为 $35 \%$. 对于卵石和砾石等球状骨料, 采用 Walraven和 Reinhardt ${ }^{[21]}$ 公式计算䈨筋混凝土柱数值模 型内部骨料的颗粒数, 见表1所示.

基于Monte-Carlo方法采用Fortran编程对表1中骨 料颗粒进行随机投放, 并将有限元网格投影到骨料结 构上. 网格单元尺寸的大小影响细观组分的准确描述 及运算效率, 依据文献[22], 混凝土细观力学分析时, 为得到稳定的宏观力学特性, 随机骨料细观力学模型 的网格单元尺寸不宜大于骨料粒径的 $1 / 4$. 本文中最小 骨料粒径为 $16 \mathrm{~mm}$, 故选用 $4 \mathrm{~mm}$ 网格单元尺寸, 4 种截 面尺寸下细观力学模型的网格单元数分别为: 897800 , 3000000, 10125000, 24000000.

表 1 钢筋混凝土柱数值模型的骨料颗粒数

\begin{tabular}{rccc}
\hline 试件 & 边长 $(\mathrm{mm})$ & 小石 $($ 粒) & 中石 $($ 粒) \\
\hline 方试件 & 150 & 18334 & 3068 \\
轴压柱 & 267 & 311079 & 51846 \\
轴压柱 & 400 & 1047272 & 174545 \\
轴压柱 & 600 & 3534545 & 589090 \\
轴压柱 & 800 & 8378181 & 1396363 \\
\hline
\end{tabular}


“界面过渡区”是骨料周围近场砂浆内孔隙率渐变 的一个区域, 其弹性模量及强度等力学性质也是渐变 的, 过渡区厚度没有严格的定义 ${ }^{[23]}$. 在进行数值模拟 时, 由于计算效率问题, 难以选取“微米”量级的单元尺 度作为混凝土有限元细观计算分析的尺度. 采用较大 的厚度(如 2 或 $4 \mathrm{~mm}$ ) 作为界面相厚度对混凝土进行力 学分析时, 若界面力学参数选取准确, 数值模拟结果 不会影响混凝土试件整体的宏观力学特性 ${ }^{[24]}$, 故本文 界面相厚度选取为 $4 \mathrm{~mm}$. 有限元网格划分后根据骨料 在网格中的位置判定单元类型, 如: 骨料单元、砂浆 单元及界面单元, 并依单元类型赋予相应的材料特性.

为揭示箍筋约束混凝土方柱轴压破坏尺寸效应, 按照1:1.5:2.25的截面尺寸比例设计与试验试件 ${ }^{[11,12]}$ 尺 寸相同的 3 组 $3 \mathrm{D}$ 约束混凝土方柱数值模型, 即截面边长 为 267,400 和 $600 \mathrm{~mm}$, 柱高为 800,1200 和 $1800 \mathrm{~mm}$, 另 外扩展构建更大尺寸 (边长为 $800 \mathrm{~mm}$, 高为 $2400 \mathrm{~mm}$ ) 的箍筋约束混凝土方柱, 其细观结构如图1(a) (d) 所示. 图1(d)中从左到右依次是混凝土方柱模型透视图、模 型内骨料颗粒分布及方柱模型的剖面构造.

体积配筪率是影响䈨筋约束混凝土柱的重要力学 指标, 依据该指标, 本文共建立3种箍筋约束混凝土方柱 数值模型, 即PA系列 $\left(\rho_{\mathrm{sv}}=0 \%\right), \mathrm{PB}$ 系列 $\left(\rho_{\mathrm{sv}}=1.26 \%\right)$ 和PC 系列 $\left(\rho_{\mathrm{sv}}=2.89 \%\right)$, 其几何参数及配筋情况如表 2 和图 2 所 示, $\mathrm{PA}$ 系列为素混凝土方柱, 构件尺寸同 $\mathrm{PB}, \mathrm{PC}$ 系列模 型. 约束混凝土柱仅在四角布置与筢筋同等直径的纵 筋, 其配筋率为 $0.28 \%$. 表中符号“P”代表棱柱体, “A”, “B”和“ $C$ ”表示体积配箍率, 分别为 $0 \%, 1.26 \%$ 和 $2.89 \%$. 试件编号中的“S”, “M”, “L”和“U”分别代表小、中、 大和超大 4 种构件尺寸. 为了防止加载过程中试件端
部过早破坏, 试件上下端各 $1 / 6$ 全高范围内, 对箍筋进 行加密布置, 约束混凝土柱的长细比 $\lambda$ 均为 3 .

\section{2 材料模型及细观组分参数}

箍等约束混凝土柱轴心加载试验采用 $\mathrm{C} 30$ 混凝土, 纵筋和䈨筋均采用HRB400级钢筋 ${ }^{[11,12]}$. 有限元细观数

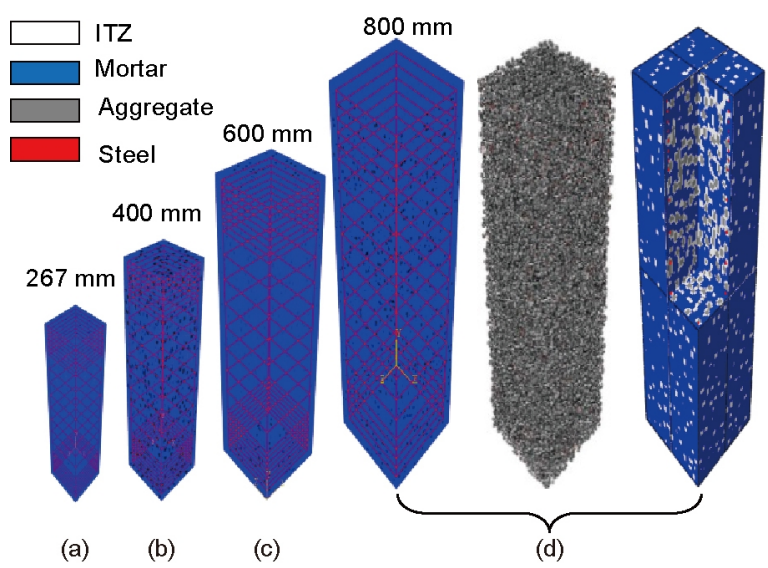

图 1 (网络版彩图)约束混凝土柱细观尺度数值模型

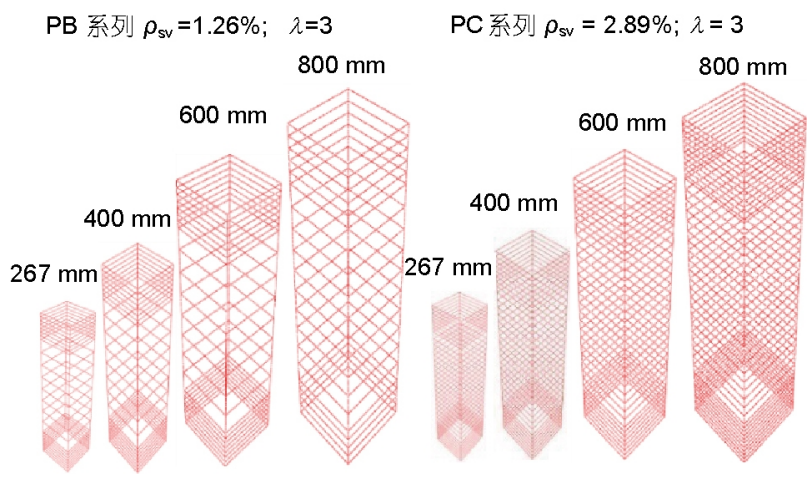

图 2 (网络版彩图)约束混凝土柱钢筋配置

表 2 约束混凝土柱几何参数与钢筋配置

\begin{tabular}{|c|c|c|c|c|c|c|}
\hline 系列 & 样本编号 & 截面尺寸 (mm) & 端区箍筋 & 中部䇥筋 & 䇥筋率 $(\%)$ & 保护层 (mm) \\
\hline \multirow{4}{*}{$\mathrm{PB}$} & PB-S & 267 & \$8@18(2) & $\$ 8 @ 66(2)$ & 1.26 & 10 \\
\hline & PB-M & 400 & $\Phi 12 @ 27(2)$ & $\$ 12 @ 99(2)$ & 1.26 & 20 \\
\hline & PB-L & 600 & $\Phi 18 @ 40(2)$ & $\Phi 18 @ 149(2)$ & 1.26 & 30 \\
\hline & PB-U & 800 & \$22@80(2) & $\$ 22 @ 160(2)$ & 1.26 & 40 \\
\hline \multirow{4}{*}{$\mathrm{PC}$} & PC-S & 267 & $\Phi 8 @ 18(2)$ & $\Phi 8 @ 29(2)$ & 2.89 & 10 \\
\hline & PC-M & 400 & $\Phi_{12 @ 27(2)}$ & $\Phi_{12 @ 44(2)}$ & 2.89 & 20 \\
\hline & PC-L & 600 & $\Phi 18 @ 40(2)$ & $\Phi 18 @ 66(2)$ & 2.89 & 30 \\
\hline & PC-U & 800 & $\Phi 22 @ 37(2)$ & $\Phi 22 @ 74(2)$ & 2.89 & 40 \\
\hline
\end{tabular}


值研究时假定混凝土由骨料颗粒、砂浆基质和界面区 组成, 同文献 $[25,26]$, 砂浆基质和界面区采用塑性损伤 本构来描述其力学性能; 骨料颗粒强度较高, 故假定不 破坏, 为弹性体. 钢筋材料力学性能较均匀, 采用理想 弹塑性本构描述其单轴应力-应变关系, 将Von-Mises 屈服面作为判断钢材屈服准则. 混凝土细观组分及钢 筋的主要力学参数见表 3 . 需要说明的是, 在骨料、砂 浆强度一定, 本文假定混凝土抗压强度只同界面力学 特性有关. 界面区的泊松比参考文献 $[27,28]$ 选取为 0.2 , 抗压强度与抗拉强度之比参考文献[29]取为 11 , 界面 区的弹性模量及单轴抗压强度数值参考混凝土材料 力学性能数值研究实践 ${ }^{[24 ~ 27]}$ 的取值范围, 通过立方体 试件(边长为 $150 \mathrm{~mm}$, 如图 3 所示) 数值反演的方式来 确定. 通过反复试算, 发现采用表 3 所示的混凝土各细 观组分时, 数值模拟结果与试验结果吻合. 该力学参
数下, 获得的混凝土单轴压缩强度为 $42.6 \mathrm{MPa}$ (与试验 测得的 $42.8 \mathrm{MPa}$ 接近), 界面区力学参数(材料弹性模 量和抗压强度)具体试算过程见表4所示.

\section{3 钢筋/混凝土细观单元相互作用}

由于钢筋/混凝土相互作用力学行为的高度复杂 性, 目前尚未得到统一的理论与力学模型, 这里为了考
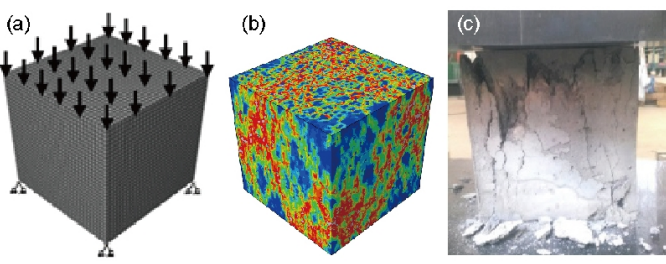

图 3 (网络版彩图)立方体试件数值模拟与试验破坏形态 对比. (a) 加载方式; (b) 压缩破坏云图; (c) 试验破坏形态

表 3 混凝土细观组分及钢筋力学参数

\begin{tabular}{ccccc}
\hline 细观组分 & 骨料 & 砂浆基质 & 界面过渡区 & 钢筋 \\
\hline 抗压强度 $(\mathrm{MPa})$ & - & $* 45.3$ & $\wedge 37.2$ & - \\
抗拉强度 $(\mathrm{MPa})$ & - & $* 6.8$ & $\wedge 3.3$ & $\wedge 24.7$ \\
弹性模量 $(\mathrm{GPa})$ & $* 60$ & $* 30.8$ & ${ }^{\circ} 0.2$ & $* 207$ \\
泊松比 & $* 0.2$ & $* 0.2$ & - & $* 0.3$ \\
屈服强度 $(\mathrm{MPa})$ & - & - & $* 477$ \\
\hline
\end{tabular}

注: “州示的力学参数为实测数据, “””所示的界面泊松比参数为参考文献所取数据, “^”所示的界面力学参数(材料弹性模量和抗压 强度)为反复试算获得的选取值.

表 4 界面区力学参数试算过程

\begin{tabular}{|c|c|c|c|}
\hline 泊松比 & 弹性模量 $(\mathrm{GPa})$ & 拉压强度度输入值 $(\mathrm{MPa})$ & 混凝土抗压强度模拟值 (MPa) \\
\hline \multirow{12}{*}{0.2} & 24.0 & $35 / 3.2$ & 40.23 \\
\hline & & $37.2 / 3.3$ & 41.01 \\
\hline & & $40 / 3.6$ & 41.68 \\
\hline & 24.4 & $35 / 3.2$ & 40.80 \\
\hline & & $37.2 / 3.3$ & 41.41 \\
\hline & & $40 / 3.6$ & 42.02 \\
\hline & 24.7 & $35 / 3.2$ & 41.17 \\
\hline & & $37.2 / 3.3$ & 42.60 \\
\hline & & $40 / 3.6$ & 43.10 \\
\hline & 25.0 & $35 / 3.2$ & 42.20 \\
\hline & & $37.2 / 3.3$ & 43.08 \\
\hline & & $40 / 3.6$ & 43.26 \\
\hline
\end{tabular}


虑钢筋与混凝土之间的黏结滑移等因素对钢筋混凝 土柱力学性能的影响, 采用我国混凝土结构设计规范 (GB50010-2010) 推荐的钢筋-混凝土黏结滑移本构关 系模型来描述其相互作用问题, 即首先将钢筋(采用 梁单元进行离散)嵌入到混凝土细观单元(采用六面体 八节点线性等参单元进行离散), 进而在钢筋单元与 混凝土细观单元间设置非线性弹簧单元, 如图4(a)所 示, 并采用如图4(b) 所示的 $\tau-S$ 曲线作为判定钢筋与混 凝土细观单元间黏结应力随滑移量变化的度量标准, 本构模型中各参数参考杜修力等人 ${ }^{[30]}$ 试验中的取值, 如表5所示, 这里需要说明的是, 该本构关系模型仅考 虑了钢筋与混凝土细观单元材料之间的切向滑移, 即 切线方向可以产生位移, 法向则认为钢筋与混凝土细 观单元间的交界面刚度无穷大, 不考虑其法向位移. 钢筋混凝土柱 $3 \mathrm{D}$ 细观力学分析模型建立后, 将其导入 ABAQUS商业软件, 进而基于ABAQUS非线性数值计 算方法来求解筷筋约束混凝土柱的宏观力学行为.

\section{3 约束混凝土柱轴心受压力学行为}

\section{1 破坏模式}

基于提出的细观数值模拟方法, 对 3 种体积配箍 率 $\rho_{\mathrm{sv}}=0 \%, \rho_{\mathrm{sv}}=1.26 \%, \rho_{\mathrm{sv}}=2.89 \%, 4$ 组不同尺寸约束混 凝土方柱的轴压破坏行为进行模拟, 柱底边固定, 在 其顶部施加位移荷载, 两侧为自由边界. 每组尺寸构 件模型数量为 2 , 各模型中骨料的体积分数相同, 仅空 间随机分布不同. 图 5 为边长尺寸为 $400 \mathrm{~mm}$ 约束混凝 土柱的轴压破坏模拟结果与试验破坏模式的对比. 从 柱子损伤变化过程(图5(a)) 可以看出, 损伤破坏首先发 生在混凝土内部的薄弱环节, 即骨料颗粒与砂浆基质 间的界面过渡区, 随着荷载增大, 损伤破坏区域向砂 浆基质延伸. 且损伤区域集中在柱中位置, 随着荷载 增加, 混凝土受压损伤区域扩大, 呈压-剪破坏形态, 材
料的非均质性导致损伤区域分布呈非均匀状态. 混凝 土柱横向膨胀过程中, 笽筋约束混凝土横向形变的作 用开始发挥, 箱筋应变不断增大, 部分箱筋达到屈服 强度, 如图 5(b) 所示. 图5(a)和(b)与试验结果图5(c) 的 对比可知, 本文数值模拟结果较好地表征了约束混凝 土柱轴心加载下的破坏行为.

数值模拟获得的 4 根几何相似, 具有不同截面尺 寸的约束混凝土方柱的最终破坏模式见图6所示, 可 以发现构件截面尺寸小于 $400 \mathrm{~mm}$ 时, 约束混凝土柱中 主要产生轴向压缩破坏; 而对于截面尺寸较大的约束 混凝土柱(截面尺寸为 600 和 $800 \mathrm{~mm}$ ), 则发生典型的 压-剪破坏形式, 这与约束混凝土构件试验结果 ${ }^{[10,11]}$ 亦 吻合一致.
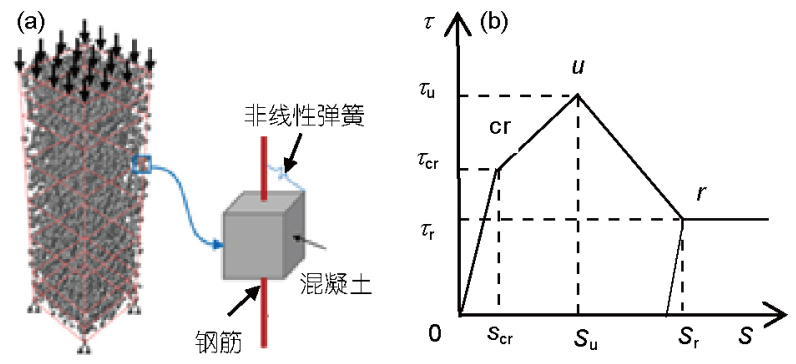

图 4 (网络版彩图)钢筋混凝土柱3D细观力学分析模型. (a) 钢筋/混凝土相互作用模型; (b) 钢筋/混凝土黏结滑移本构

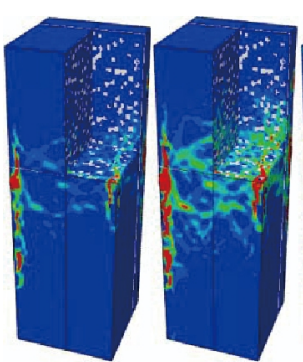

(a)

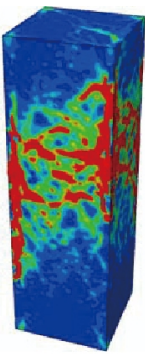

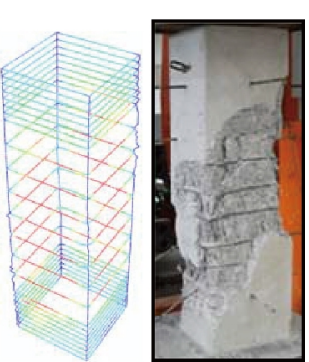

(b)

(c)
图 5 (网路版彩图)约束混凝土柱(边长 $400 \mathrm{~mm}$ )损伤破坏. (a) 混凝土柱损伤演化; (b) 钢筋笼反应; (c) 试验结果

表 5 混凝土细观单元与钢筋间粘结应力-滑移曲线的参数值 ${ }^{a}$

\begin{tabular}{|c|c|c|c|}
\hline 特征点 & 䢃裂(cr) & 峰值 (u) & 残余 (r) \\
\hline 黍结应力 $\tau(\mathrm{MPa})$ & $\tau_{\mathrm{cr}}=2.5 f_{\mathrm{t}}$ & $\tau_{\mathrm{u}}=3 f_{\mathrm{t}}$ & $\tau_{\mathrm{r}}=f_{\mathrm{t}}$ \\
\hline 纵筋相对滑移 $S_{1}(\mathrm{~mm})$ & $S_{\mathrm{cr}, 1}=0.025 d_{1}$ & $S_{\mathrm{u}, 1}=0.04 d_{1}$ & $S_{\mathrm{r}, \mathrm{I}}=0.55 d_{1}$ \\
\hline 箍筋相对滑移 $S_{\mathrm{t}}(\mathrm{mm})$ & $S_{\mathrm{cr}, \mathrm{t}}=0.025 d_{\mathrm{t}}$ & $S_{\mathrm{u}, \mathrm{t}}=0.04 d_{\mathrm{t}}$ & $S_{\mathrm{r}, \mathrm{t}}=0.55 d_{\mathrm{t}}$ \\
\hline
\end{tabular}

a) “ $f$ ””为实测混凝土抗拉强度, “ $d$ ”为纵筋直径, “ $d \mathrm{t}$ ”为䈐筋直径. 


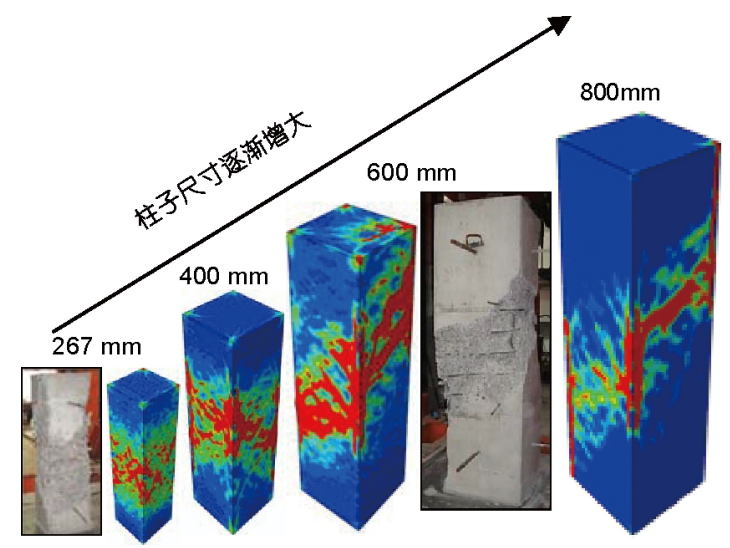

图 6 (网络版彩图) 不同尺寸约束混凝土柱的破坏模式

\section{2 名义轴压强度与应力应变关系曲线}

数值模拟获得的名义轴压强度见表 6 . 可见, 数 值模拟与试验结果吻合很好 (误差不超过 $5 \%$ ), 再次
证实了数值方法的有效性. 另外, 扩展模拟得到素混 凝土柱及更大尺寸 (边长 $800 \mathrm{~mm}$ ) 配箍率 $1.26 \%, 2.89 \%$ 方柱名义轴压强度均值为 $30.07,38.52,42.16 \mathrm{MPa}$. 随 着配䈐率的增加, 4 种尺寸方柱的名义轴压强度有所 提高. 相同体积配䇚率下, $\mathrm{PA}\left(\rho_{\mathrm{sv}}=0 \%\right), \operatorname{PB}\left(\rho_{\mathrm{sv}}=1.26 \%\right)$, $\mathrm{PC}\left(\rho_{\mathrm{sv}}=2.89 \%\right) 3$ 种系列构件模型的名义轴心抗压强度 均随着构件尺寸的增大而降低, 表明约束混凝土方柱 存在尺寸效应现象.

尺寸为 $267 \mathrm{~mm} \times 267 \mathrm{~mm} \times 800 \mathrm{~mm}, 400 \mathrm{~mm} \times 400$ $\mathrm{mm} \times 1200 \mathrm{~mm}$, 体积配箱率为 $0 \%, 1.26 \%, 2.89 \%$ 约束混 凝土方柱数值模拟得到的应力应变关系曲线见图7所 示. 图中横坐标是柱端坚向位移 $\Delta$ 与柱高 $H$ 的比值, 纵坐标轴向应力为轴向荷载与柱横截面面积的比值. 由图7可见, 由于核心区混凝土处于三轴受压应力状 态, PB, PC系列约束混凝土构件模型的轴压强度和变 形能力均较单轴受压的 PA系列素混凝土柱有较大提

表 6 数值模拟和试验结果对比

\begin{tabular}{|c|c|c|c|c|}
\hline 样本编号 & 数值解 $(\mathrm{MPa})$ & 数值解均值 (MPa) & 试验结果 (MPa) & 误差 $(\%)$ \\
\hline PA-S-1 & 39.86 & 40.21 & - & - \\
\hline PA-S-2 & 40.56 & & & \\
\hline PA-M-1 & 35.05 & 35.64 & - & - \\
\hline PA-M-2 & 36.22 & & & \\
\hline PA-L-1 & 33.06 & 32.45 & - & - \\
\hline PA-L-2 & 31.83 & & & \\
\hline PA-U-1 & 29.50 & 30.07 & - & - \\
\hline PA-U-2 & 30.64 & & & \\
\hline PB-S-1 & 45.22 & 46.12 & 47.63 & 3.17 \\
\hline PB-S-2 & 47.03 & & & \\
\hline PB-M-1 & 43.27 & 43.99 & 43.47 & 1.19 \\
\hline PB-M-2 & 44.71 & & & \\
\hline PB-L-1 & 41.33 & 42.04 & 42.60 & 1.30 \\
\hline PB-L-2 & 42.75 & & & \\
\hline PB-U-1 & 39.17 & 38.52 & - & - \\
\hline PB-U-2 & 37.86 & & & \\
\hline PC-S-1 & 48.03 & 48.99 & 49.80 & 1.63 \\
\hline PC-S-2 & 49.95 & & & \\
\hline PC-M-1 & 47.27 & 47.40 & 47.99 & 1.23 \\
\hline PC-M-2 & 47.53 & & & \\
\hline PC-L-1 & 47.06 & 46.88 & 47.23 & 0.67 \\
\hline PC-L-2 & 46.70 & & & \\
\hline PC-U-1 & 42.03 & 42.16 & - & - \\
\hline PC-U-2 & 42.29 & & & \\
\hline
\end{tabular}



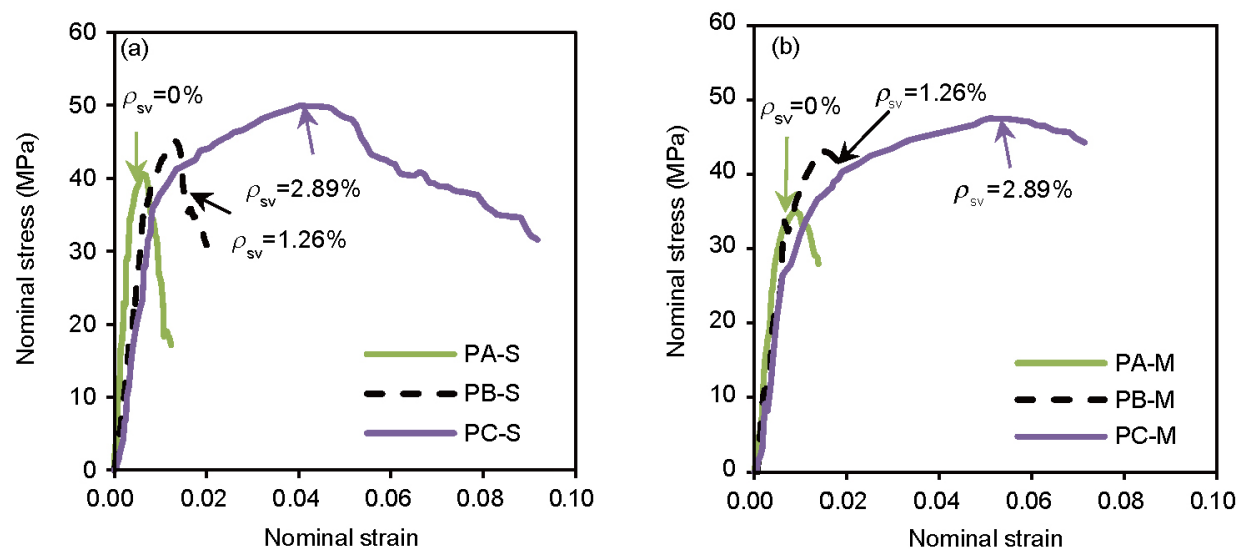

图 7 (网络版彩图)约束混凝土方柱应力应变曲线. (a) $267 \mathrm{~mm} \times 267 \mathrm{~mm} \times 800 \mathrm{~mm}$ 模型; (b) $400 \mathrm{~mm} \times 400 \mathrm{~mm} \times 1200 \mathrm{~mm}$ 模型

高. 特别是构件模型达到峰值强度后, 混凝土材料横 向膨胀量明显增大, 箱筋的约束作用随之增强. 在䈨 筋的约束作用下, 核心区混凝土的膨胀变形和剪切变 形被限制, 因而名义轴压强度和变形能力显著提高, 体积配箱率越大, 提高的幅度越大. 体积配筢率 $2.89 \%$ 的PC系列构件模型的应力应变曲线软化段名义强度 缓慢降低, 破坏具有较大的塑性

数值模拟与试验结果的良好吻合证明了数值方 法的有效性. 后续工作中, 将基于该套数值分析方法, 开展系列的参数分析, 如探讨更多配䈨率、柱子截面 形式、钢筋屈服强度及混凝土强度等级等对䇚筋约 束混凝土柱尺寸效应的定量影响.

\section{4 约束混凝土轴心抗压强度尺寸效应分析}

\section{1 名义轴压强度与构件尺寸关系}

箍筋约束混凝土柱数值模拟的名义轴压强度 $\sigma_{\mathrm{Nu}}$ 随构件尺寸 $D$ (即柱子截面边长) 的变化规律如图 8 所 示, 可见: (1) 三组柱子的名义轴压强度均随构件尺寸 的增加而降低, 即存在尺寸效应现象; (2) 配䈐率越大, 约束作用越强, 柱子名义轴压强度越大; (3) 配䈨率越 大, 名义强度随尺寸增大而减小的幅度越小, 即强度 减弱越缓慢. 相比于PC系列, PB系列柱的体积配䈨率 低, 约束作用弱, 破坏具有更强的脆性, 因而表现出更 明显的尺寸效应. PA系列素混凝土柱轴压受压破坏的 脆性最大, 因而尺寸效应现象最明显.

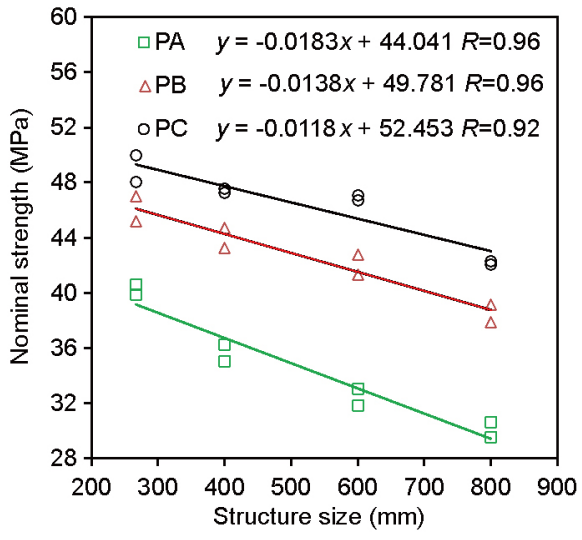

图 8 (网络版彩图)约束混凝土柱强度尺寸效应曲线

\section{2 约束混凝土柱强度尺寸效应理论分析}

基于断裂力学理论, Bažant提出了适用于混凝土 材料及钢筋混凝土构件的尺寸效应理论公式 ${ }^{[31]}$ :

$\sigma_{\mathrm{Nu}}=\frac{B f_{t}^{\prime}}{\sqrt{1+\beta}}, \quad \beta=D / D_{0}$,

式中, $\sigma_{\mathrm{Nu}}$ 为名义强度, $f_{\mathrm{t}}^{\prime}$ 为混凝土材料䢃裂抗拉强度, $B, D_{0}$ 是依赖于结构的几何常数 (由模拟数据拟合获得), $D$ 是试件尺寸参数(这里为截面边长), $\beta$ 是反映材料脆 性大小的参数. 根据数值实验结果, 通过回归分析来 确定常数项 $B f_{\mathrm{t}}^{\prime}$ 和 $D_{0}$. 式(1)可表达成线性方程:

$y=A x+C$,

式中, $x=D, y=1 / \sigma_{\mathrm{N}_{\mathrm{u}}}^{2}, C=1 /\left(B f_{t}^{\prime}\right)^{2}, A=C / D_{0}$. 通过 回归法求解得到线性方程见图9所示, 由图中线性方 
程的系数得到Bažant尺寸效应律拟合参数如表7所示, 进而可以绘制尺寸效应理论拟合曲线(图10).

由表7可见, $\mathrm{PA}$ 系列素混凝土方柱的脆性参数 $\beta$ 最大, $\mathrm{PC}$ 系列约束混凝土方柱模型的脆性参数 $\beta$ 最小. 表明体积配䈨筋越高, 约束混凝土方柱轴压破坏的脆 性越小. 图10中约束混凝土柱数值模拟结果与Bažant 尺寸效应理论拟合方程的相关系数为 0.93 , 说明数值 模拟结果与Bažant尺寸效应理论吻合良好. 可以看出: 素混凝土柱模型(PA系列)的名义强度数据点最趋近 LEFM, 即斜率为 $-1 / 2$ 的直线, 体积配箍率越大, 强度 数据点越远离该直线, 更趋近于斜率为零的水平渐近 线. 简言之, 体积配笼率越小, 约束混凝土柱模型破坏 脆性越大, 名义轴压强度下降越为明显, 表现出更为 明显的尺寸效应现象。

\section{5 结论}

本文采用钢筋混凝土柱轴压破坏行为及尺寸效 应模拟的 $3 \mathrm{D}$ 细观尺度数值分析方法, 开展了体积配䇽 为 $0 \%, 1.26 \%$ 和 $2.89 \%$, 不同截面尺寸箍筋约束混凝土 方柱轴心加载数值实验, 探讨了䈨筋约束作用对钢筋 混凝土方柱轴压破坏模式及尺寸效应的影响规律. 并 与试验结果对比验证分析, 在模拟结果与试验结果吻 合良好的基础上, 扩展模拟了更大尺寸方柱的破坏行 为. 同时结合Bažant尺寸效应理论开展了䈐筋约束混 凝土柱轴心抗压强度尺寸效应理论分析, 主要研究结 论如下.

(1) 构建的钢筋混凝土柱 $3 \mathrm{D}$ 细观分析模型较好地 反映了䈨筋约束混凝土柱轴心加载下的损伤破坏行 为, 即: 损伤主要发生在薄弱的界面区域并向砂浆基 质扩展, 由于材料的非均质性导致损伤区域分布呈非 均匀状态。

(2) 数值模拟与试验结果吻合良好, 钢筋混凝土柱 3D细观分析模型很好地描述了几何尺寸对构件破坏 行为以及名义强度的影响, 可为约束混凝土构件尺寸
效应研究提供理论支持.

(3) 体积配䈨率越大, 核心区混凝土受到的箱筋约 束作用越强, 钢筋混凝土方柱的名义轴压强度数值越 大, 构件破坏时呈现出更少的脆性, 表现出更弱的尺 寸效应现象。

(4) Bažant尺寸效应理论能够很好地描述箍筋约 束混凝土方柱在轴心加载下的破坏行为及尺寸效应 规律.

本文钢筋/混凝土细观单元本构关系模型仅考虑

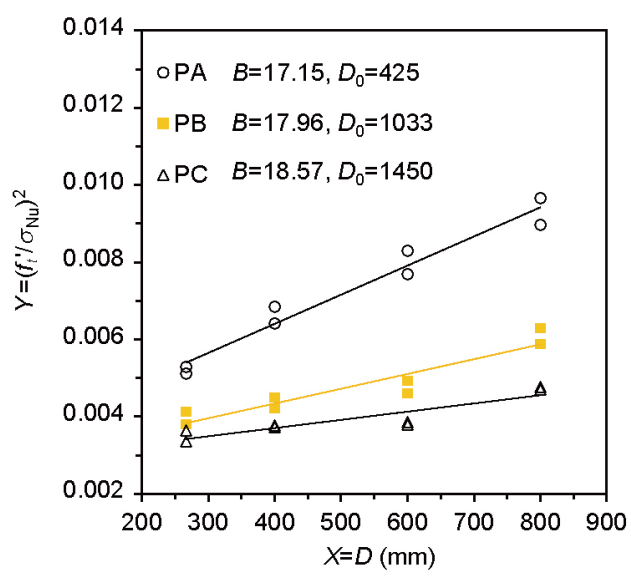

图 9 (网络版彩图)Bažant尺寸效应参数拟合方程

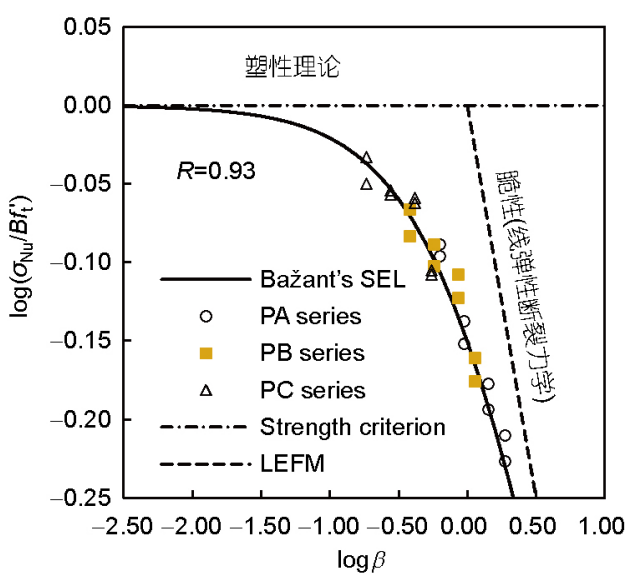

图 10 (网络版彩图)Bažant尺寸效应理论拟合曲线

表 7 Bažant尺寸效应理论参数

\begin{tabular}{cccccc}
\hline 系列编号 & $A\left(\times 10^{-5}\right)$ & $C\left(\times 10^{-2}\right)$ & $B f^{\prime}{ }_{\mathrm{t}}(\mathrm{MPa})$ & $D_{0}(\mathrm{~mm})$ & $\beta$ \\
\hline PA & 0.80 & 0.34 & 49.74 & 425 & 0.63 \\
$\mathrm{~PB}$ & 0.30 & 0.31 & 52.08 & 1033 & 0.38 \\
$\mathrm{PC}$ & 0.20 & 0.29 & 53.85 & 1450 & 0.18 \\
\hline
\end{tabular}


了钢筋与混凝土细观单元材料之间的切向滑移, 未考 虑其法向位移, 存在不足. 鉴于此, 建立能够准确表征 钢筋/混凝土细观单元间相互作用及尺寸效应的三维 细观尺度数值模型, 在与试验结果吻合良好的基础上
开展大量箱筋约束混凝土柱数值模拟工作, 探索研究 不同长细比、体积配箍率和强度等级以及复合受力 状态下箍筋约束混凝土柱尺寸效应行为, 是今后研究 工作的重点和难点问题.

\section{参考文献}

1 史庆轩, 侯炜, 张兴虎, 等. 箍筋约束混凝土结构及其发展展望. 建筑结构学报, 2009, 30: 109-114

2 De Diego A, Arteaga A, Fernández J, et al. Behaviour of FRP confined concrete in square columns. Mater Construcc, 2015, 65 : e069

3 Richart F E, Brandtzaeg A, Brown L R. A study of the failure of concrete under combined compressive stresses. Technical Reports. Illinois: Urbana University of Illinois, 1928. 82-95

4 Soliman M T M, Yu C W. The flexural stress-strain relationship of concrete confined by rectangular transverse reinforcement. Mag Concrete Res, 1967, 19: 223-238

5 Mander J B, Priestley M J N, Park R. Observed stress-strain behavior of confined concrete. J Struct Eng, 1988, 114: 1827-1849

6 钱稼茹, 程丽荣, 周栋梁. 普通筢筋约束混凝土柱的中心受压性能. 清华大学学报, 2002, 42: 1369-1373

7 史庆轩, 杨坤, 刘维亚, 等. 高强箍筋约束高强混凝土轴心受压力学性能试验研究. 工程力学, 2012, 29: 141-149

8 郑文忠, 侯羽驰. 不同强度等级筢筋约束混凝土柱设计方法. 建筑结构学报, 2016, 37: 74-82

9 West J, Ibrahim A, Hindi R. Analytical compressive stress-strain model for high-strength concrete confined with cross-spirals. Eng Struct, 2016, 113: $362-370$

10 中国人民共和国建设部. 混凝土结构设计规范GB50010-2010. 北京: 中国建筑工业出版社, 2010

11 Li Z, Song J, Du X, et al. Size effect of confined concrete subjected to axial compression. J Cent South Univ, 2014, 21: 1217-1226

12 李振宝, 宋佳, 杜修力, 等. 方形箍筋约束混凝土力学性能尺寸效应试验研究. 北京工业大学学报, 2014, 40: 223-229

13 车轶, 王铁东, 班圣龙, 等. 䈨筋约束混凝土轴心受压性能尺寸效应研究. 建筑结构学报, 2013, 34: 118-123

14 Bažant Z P, Kwon Y W. Failure of slender and stocky reinforced concrete columns: Tests of size effect. Mater Struct, 1994, 27: 79-90

15 Nemecek J, Bittnar Z. Experimental investigation and numerical simulation of post-peak behavior and size effect of reinforced concrete columns. Mater Struct, 2004, 37: 161-169

16 Li D, Jin L, Du X L, et al. Size effect tests of normal-strength and high-strength RC columns subjected to axial compressive loading. Eng Struct, 2016, 109: 43-60

17 Rios R D, Riera J D. Size effects in the analysis of reinforced concrete structures. Eng Struct, 2004, 26: 1115-1125

18 Wang L C. Meso-scale numerical modeling of the mechanical behavior of reinforced concrete members. Int J Eng Tech, 2013, 5: 680-684

$19 \mathrm{Du}$ X L, Jin L, Ma G W. Meso-element equivalent method for the simulation of macro mechanical properties of concrete. Int J Damage Mech, 2013, 22: 617-642

20 Bažant Z P. Scaling theory for quasibrittle structural failure. Proc Natl Acad Sci USA, 2004, 101: 13400-13407

21 Walraven J C, Reinhardt H W. Theory and experiments on the mechanical behavior of cracks in plain and reinforced concrete subjected to shear loading. Heron, 1981, 26: 23-33

22 Guo L P, Carpinteri A, Roncella R, et al. Fatigue damage of high performance concrete through a 2D mesoscopic lattice model. Comput Mater Sci, 2009, 44: 1098-1106

23 陈惠苏, 孙伟, Stroeven P. 水泥基复合材料与浆体界面研究综述: 界面微观结构的形成、劣化机理及其影响因素. 硅酸盐学报, 2004, 32: $70-79$

24 杜修力, 金汶. 考虑过渡区界面影响的混凝土宏观力学性质研究. 工程力学, 2012, 29: 72-79

25 Unger J F, Eckardt S. Multiscale modeling of concrete. Arch Comput Methods Eng, 2011, 18: 341-393

26 李冬, 金汶, 杜修力, 等. 钢筋混凝土轴压柱长细比效应的细观数值研究. 中国科学: 技术科学, 2016, 46: 961-969

27 杜敏, 杜修力, 金汶, 等. 混凝土拉压强度尺寸效应的细观非均质机理. 土木建筑与环境工程, 2015, 37: 11-17

28 Jin L, Li D, Du X L. Mechanical behavior and size effect of moderate high-strength RC columns under monotonic and cyclic axial compression. Eng Struct, 2016, 124: 269-285

29 黄政宇. 土木工程材料. 北京: 高等教育出版社, 2002. 74-80

30 杜修力, 符佳, 张建伟. 钢筋混凝土柱轴心受压性能尺寸效应的大比尺试验研究. 土木工程学报, 2010,43: 1-8 
31 Hoover C G, Bažant Z P. Cohesive crack, size effect, crack band and work-of-fracture models compared to comprehensive concrete fracture tests. Int J Fract, 2014, 187: 133-143

\title{
Mesoscopic simulation of size effect on stirrup-confined concrete columns under axial compression
}

\author{
DU Min ${ }^{1,2}$, JIN Liu $^{2}$, DU XiuLi², LI Dong ${ }^{2} \&$ ZHANG Shuai ${ }^{2}$ \\ ${ }^{1}$ Department of Disaster Prevention Engineering, Institute of Disaster-prevention Science and Technology, Sanhe 065201, China; \\ ${ }^{2}$ Key Laboratory of Urban Security and Disaster Engineering, Beijing University of Technology, Beijing 100124, China
}

Size effect of reinforced concrete (RC) members is mainly caused by the heterogeneity and mutual effect of concrete and steel. Considering of the above influential factors, the 3D meso-mechanical models of stirrup-confined concrete square columns were set up and the damage process subjected to axial compressive loading was simulated. The confinement effect on the failure patterns and the axial compressive strength of the stirrup-confined columns was explored. Moreover, the simulated results were compared with test data. Based on the basic theory of the size effect law (SEL) proposed by Bažant, size effect of stirrup-confined concrete columns was analyzed. It was showed that the simulated results have a good consistence with the test data and the mesoscopic analysis models of RC columns can accurately describe the damage behavior and size effect of stirrup-confined columns. With the increase of stirrup ratio, the nominal strength and deformation behavior of columns boost. Then, it becomes little brittle in the damage process. Therefor the size effect is little obvious. The simulation data fit well with basic theory of the size effect law (SEL) proposed by Bažant.

stirrup-confined, concrete square columns, axial compressive strength, size effect, stirrup ratio, meso-scale simulation

doi: $10.1360 / \mathrm{N} 092017-00240$ 